\title{
Test Rig Design and Preliminarily Assessment for a Transradial Socket
}

\section{Bondok, MA El-Sheikh and MA El-Hadek}

Production and Mechanical Design Department, Faculty of Engineering, Port-Said University, Egypt

\begin{abstract}
Introduction: The transradial level is the most common level of upper-limb amputation seen by the prosthetist. The socket is an intermediate part to wear and fit the prosthetic device with the residual limb firmly. It is the crucial part of the prosthesis influencing the amputee's acceptance considerably. The study introduces a test rig that can assess the socket while simulating arm movements.

Methods: The selected socket and test rig designs were drawn by SolidWorks CAD software. Test rig has abilities to tilt at any plane to simulate the arm movement and made of wood. The common movements during activities of daily living are (Abduction, Adduction, Flexion, Extension, Supination and Pronation) in Euler angles (Yaw, Pitch and Roll). A live-lift and axial load tests were done by the test rig in Euler angles simulating common arm movements.

Results: The live-lift force is larger than the axial-load force at all cases.

Conclusion: This experimental work covers two main tests for assessment the transradial socket at different positions and planes. It can predict when dislocation between the socket and residual limb will happen at any weight and plane.
\end{abstract}

\section{Keywords}

Test rig, Transradial amputation, Socket, Upper limb prosthetics

\section{Introduction}

The study showed that the ratio of upper amputation to lower amputation was approximateIy 1:2.2. Upper limb amputees have an enormous sense of frustration and problems in the rehabilitation process because of technical difficulties in reproducing delicate and complex movements and the tactile and proprioceptive sensory functions [1].
The $70 \%$ of all persons with upper limb amputations have amputations distal to the elbow. Transradial amputation level occurs in the forearm, from the elbow to the wrist [2]. Amputee needs intermediate part to wear and fit the prosthetic device firmly with the residual limb. So the socket is the only channel between residual limb and prosthetic components and is the critical part of the prosthesis affecting the amputee's acceptance considerably [3].

*Corresponding author: MA El-Sheikh, Production and Mechanical Design Department, Faculty of Engineering, Port-Said University, Egypt

Accepted: January 13, 2020; Published: January 15, 2020

Copyright: (c) 2020 Bondok MM, et al. This is an open-access article distributed under the terms of the Creative Commons Attribution License, which permits unrestricted use, distribution, and reproduction in any medium, provided the original author and source are credited.

Bondok et al. Int J Robot Eng 2020, 5:020 
Assessment of transradial socket prosthetic is very important for measurement how amputees are satisfied about prosthetic interface. A survey showed that $20 \%$ of upper-limb amputees had abandoned prosthesis use. The critical factor is the comfort and functionality [4]. The comfort-related problems are such as weight, fit, and heat [5].

There are many methods and technologies to fit the sockets. A silicone suction socket technology can easily achieve true suction suspension and fitting the long transradial limb. A silicone suction socket preserves forearm rotation because it has low and flexible trim lines. For fitting long transradial, wrist disarticulation, and partial hand prostheses, two construction methods have been used (all-silicone construction and hybrid construction with silicone) [6]. The suspension was also improved and increasing of flexion range could be observed. The Netherlands team created an economical socket that was donned easily. The design of resulting frame considers anatomical structures and biomechanical principals for the structure distal to the socket [7].

Wayne K. Daly presented system that is adaptable to many amputation levels that maintains contact of electrode for patients who have volume changes or suffers from difficulty with the traditional suspension methods. Electrodes in roll-on sleeves have been tested to provide improved suspension. The Roll-on sleeve is an excellent way to accomplish superior suspension and greater range of motion [8].

The motion capture model and Slip Detection Sensor is used by ref [9]. The 3D motion capture model allows for measurement of the movement that occurs at interface of the prosthetic socket. The Slip Detection Sensor measures the amount of socket slip which occurs between the socket wall and surface of the residual limb skin. Kinematic data was collected for each participant during the reiteration of a series of range of motion included shoulder flexion/extension, shoulder rotation, and elbow flexion, shoulder abduction/adduction, and activities of daily living included a modified box and blocks test, bilateral and unilateral lifting task at various weight increments, walk and carry a gallon jug of water, and folding a towel. The effect of donning the prosthesis on the range of motion of participant and the amount of socket movement during the activities of daily living tasks was ana- lyzed [9].

On the contrary of the previous studies, Increasing the modularity is the focus of the study [10] that presented a modular upper-limb bypass socket to perform tests of prosthetic systems from the prosthetic user's perception. Developers and inventors can perform tests and make improvements with bypass sockets before formally testing with an amputee. Bypass socket accesses to forearm musculature and the hand that are necessary to provide substituted sensory feedback and allows a sufficient range of motion to accomplish tasks in the frontal working area. Tests are performed by non-amputee participants [10].

Also Liz Haverkate depended on able-bodied subjects in his assessment of body-powered upper limb prostheses to assess and compare the functional performance three body-powered upper limb terminal devices which commonly used. In this work, two functional tests; the Nine Hole Peg Test and the Box and Blocks Test are performed by able-bodied subjects [11].

The lower limbs sockets exposed many tests and assessment. H. W. Wevers in 1987 designed a dynamic testing machine incorporating preliminary standards to mimic loads under normal walking conditions except the torsion along the long axis of the limb [12]. Reference [13] evaluated two designs of mono limbs with different shapes of shank. Gait Cycle Testing provides static and dynamic gait data for lower limb amputee. This test measures barefoot pressure and force for patient [14].

Upper-Limb Socket Prosthetic is needing different tests to be assessed such as Pull-off force test, Practical Activities Test, Live-lift test, Axial-load test, and positioning control test $[8,10,15]$. There is rarity in the previous test rig designs for sockets of the upper limb. So, the goal of this study is to objectively designing and fabricating a Test Rig to assess the upper-limb socket prosthetic in Euler angles: Pitch, yaw and roll while simulating the arm movements during activities of daily living such as: Abduction and Adduction, Flexion and Extension, Supination and Pronation. Also, it allows to predict when dislocation between residual limb and the socket will be happened with any weight and at any plane.

\section{Methods}

In this design, we used the SolidWorks CAD software to make the model of the test rig. This 


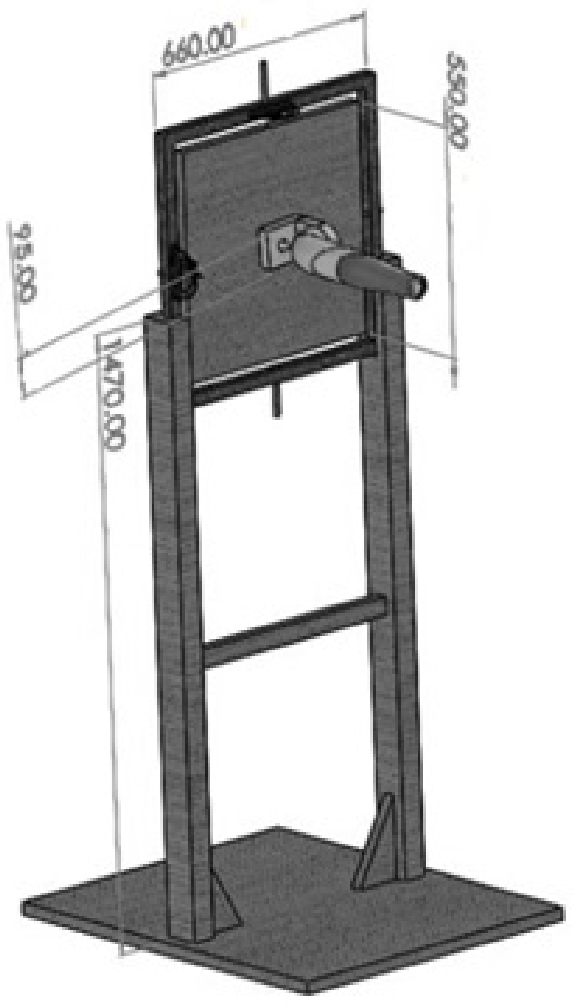

Figure 1: Test rig design (Dimensions in $\mathrm{mm}$ ). design allows to test the upper-limb prosthetic socket in Euler angles: Yaw, Pitch and Roll as shown in Figure 1. To measure at any weight with any plane the socket will be dislocated from the residual limb, The authors introduce the test rig that has ability to tilt universally at any plane.

\section{The design of the tested transradial socket}

For drawing the conventional transradial socket, the author locates marks on the socket at equal distances as shown in Figure 2a. By using a caliper, the anteroposterior and medialateral dimensions of the elliptical sections at each mark are measured. Then, the sketches of the several elliptical sections of the socket were drawn on the SolidWorks CAD software and loft feature were done.

Figure $2 \mathrm{~b}$ illustrates the below-elbow socket prosthetic that has been drawn and assessed by the study, illustrating sections of the socket at different positions. Sections $X-X$ and B-B have an elliptical shape. The elliptical shape decreases gradually to become circular shape finally.

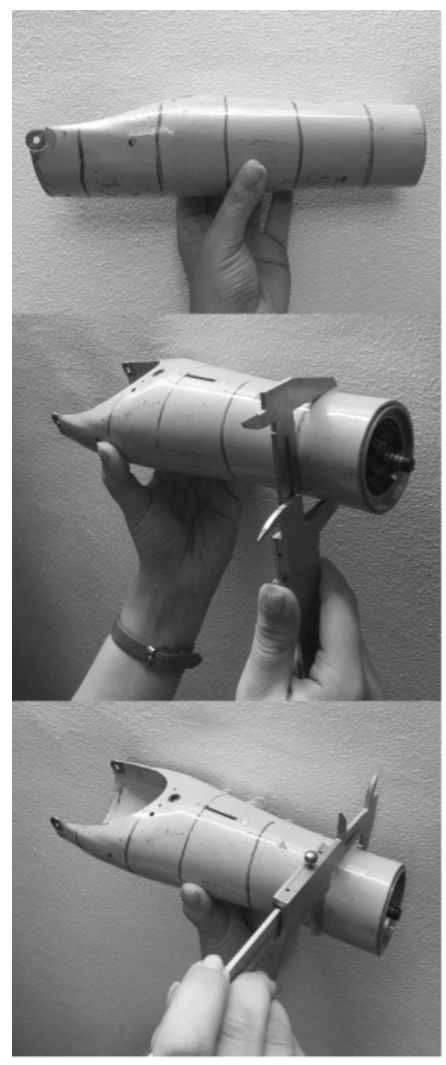

a
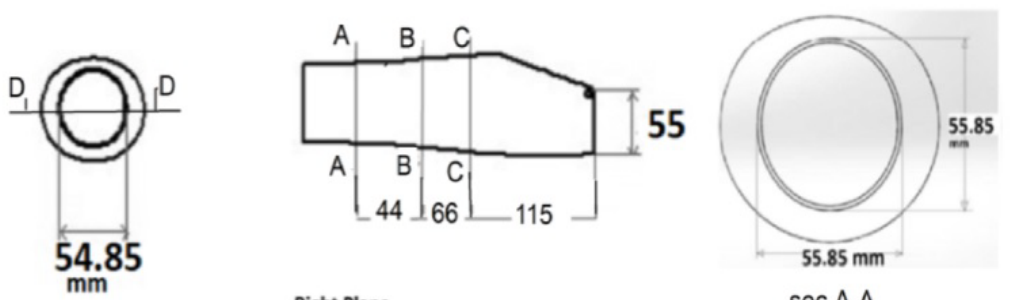

$\sec A-A$
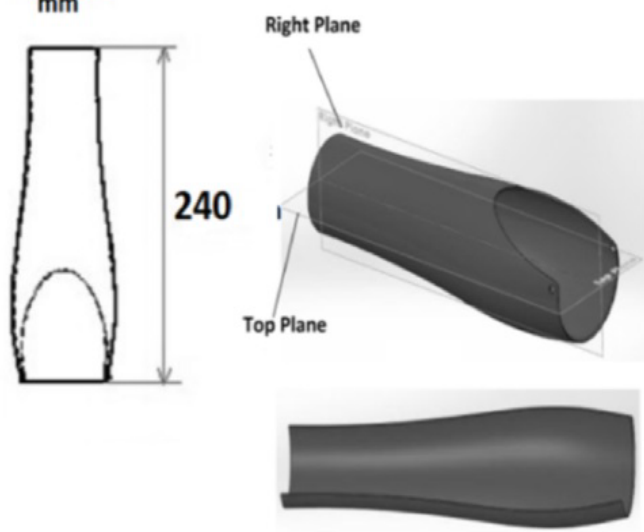

$\sec \mathrm{D}-\mathrm{D}$

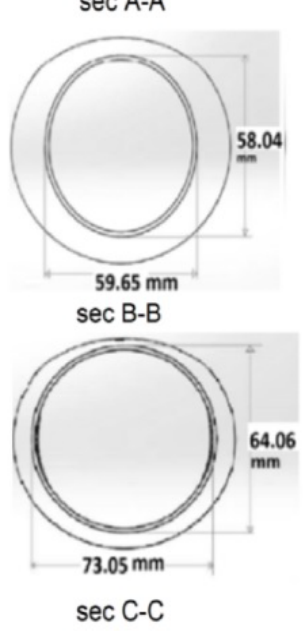

b

Figure 2: a) Marks putted on the socket and anteroposterior and medialateral dimensions of the elliptical sections were measured; $b$ ) The socket design on SolidWorks CAD software and his sections at (C-C), (B-B), (X$X)$, and (D-D). 


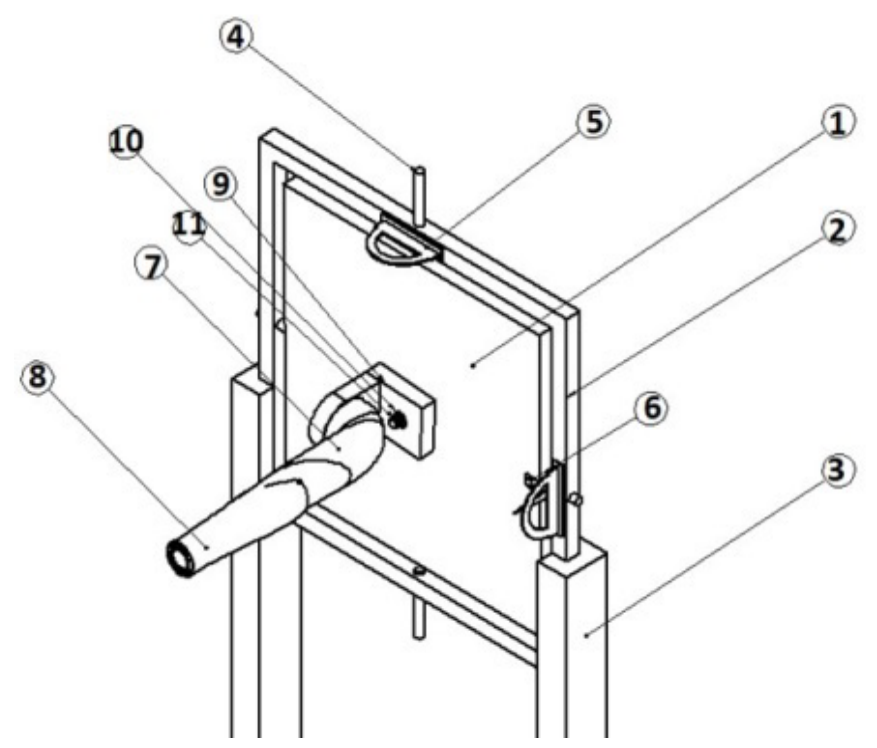

Figure 3: Parts of test rig (1- Plate, 2- Frame, 3- Plate form, 4- Wooden pin, 5- Protractor, 6- Indicator, 7- Arm, 8- Socket, 9- connection part, 10- Nut, 11- Pin stud).

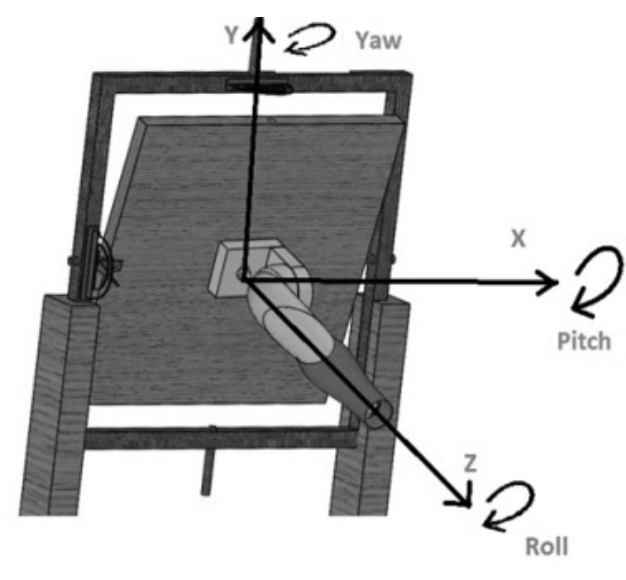

a

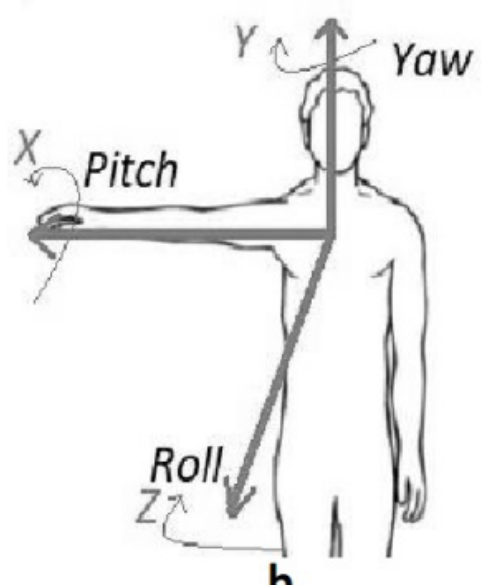

b

Figure 4: a) Represent of (Pitch, Yaw and Roll angles) on the body human; b) Represent of (Pitch, Yaw and Roll angles) on the test rig.

\section{Design and fabrication process for the test rig}

The used material to make this design is wood. The design consists of main parts as: Square plate $55 \mathrm{~cm} \times 55 \mathrm{~cm}$ and square frame $66 \mathrm{~cm} \times 66 \mathrm{~cm}$ and both of them with $3 \mathrm{~cm}$ in thickness, plat form with $1470 \mathrm{~mm}$ in height (Item no. 3), connection part between plate and arm (Item no. 9), four wooden pins (Item no. 4), two protractors (Item no. 5), and two Indicators (Item no. 6) as shown in Figure 3.

The purpose of this study is making a test rig that can assess the socket while simulating the arm movements, Abduction and Adduction, Flexion and Extension, Supination and Pronation are common during activities of daily living. All of these movements can be represented through this design in Euler angles: Pitch, yaw and roll as shown in Figure 4.

The design has been made to simulate the movements of Euler angles by supplying it with four wooden pins and connection part between plate and the arm as shown in Figure 5a. Rotating about $X$ and $Y$ axis to simulate pitch and yaw movements are shown in Figure $5 b$ and Figure 5c. Releasing the connection part between the plate and the arm makes the socket to simulate the roll movement as shown in Figure $5 \mathrm{~d}$. 

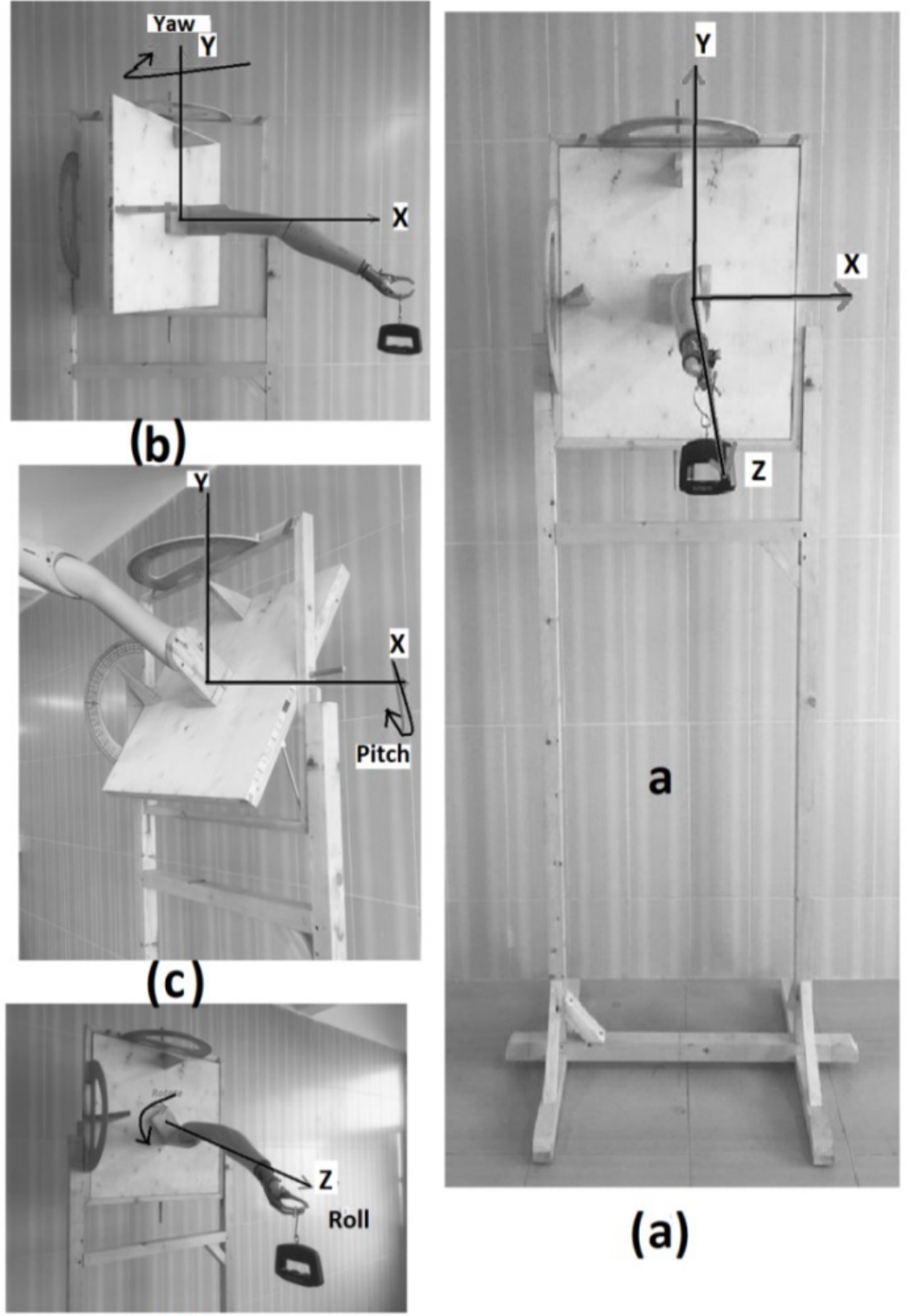

(d)

(a)

Figure 5: a) Design and fabricating (The test rig); b) Pitch angle by rotation about the $Y$ axis $Y$-axis; $c$ ) Yaw angle by rotation about the $\mathrm{X}$-axis; and d) Roll angle by rotation about the Z-axis.

The abduction and adduction are used to describe the movements that are towards or away from the midline of the body. The horizontal abduction: For the start position, arm is lifted in front of the body. The action occurs when arm moved out to the side. The horizontal adduction: From the starting position, when arm is lifted out to the side of the body. The actions occurs when arm is moving in front of the body as shown in Figure 6a.

The flexion and extension movements are used to describe increasing and decreasing the angle between two body parts and occurred in the sagittal plane. The flexion occurs when the angle is decreased between two body parts. The extension occurs when the angle is increased between the two body parts as shown in Figure 6b.

The pronation and supination are a pair of movements that occurred at the proximal radioulnar joint. The two unique movements allow the human body to flip the palm. The supination occurs when the palm is facing upwards and the pronation when the palm is facing downwards as shown in Figure 6c.

\section{Experimental work}

The two tests were done by this test rig, a liveLift Test, and an axial load test. The two experiments were in Euler angles and simulated common 


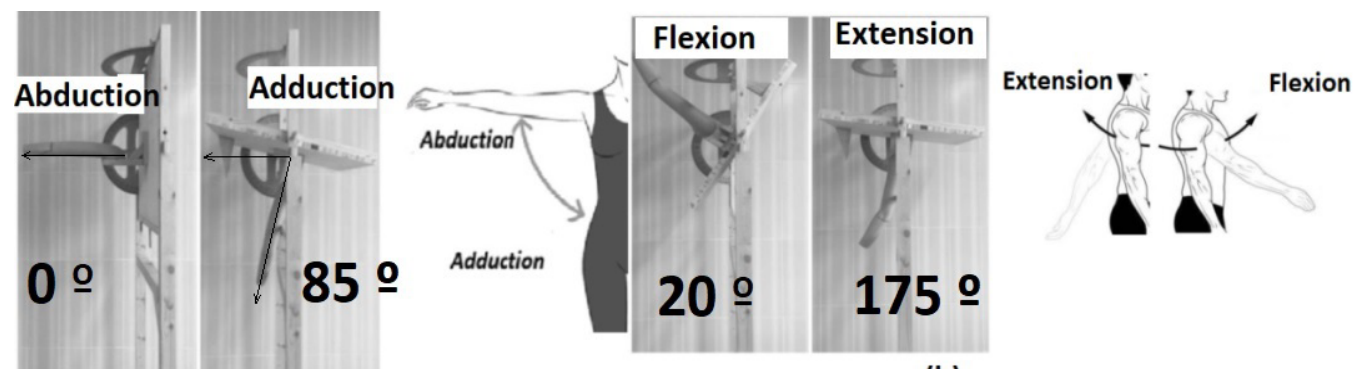

(a)

(b)

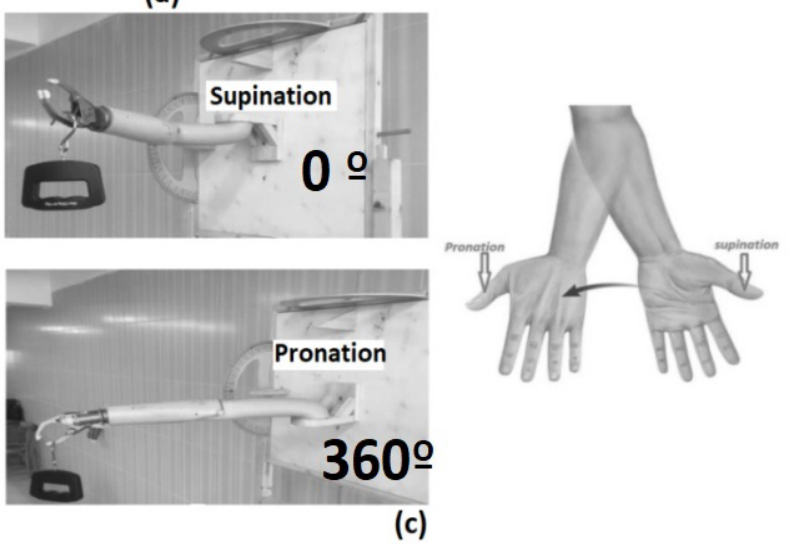

Figure 6: a) Abduction and adduction movements; b) Flexion and extension movements; and c) Supination pronation movements by test rig design and body human.

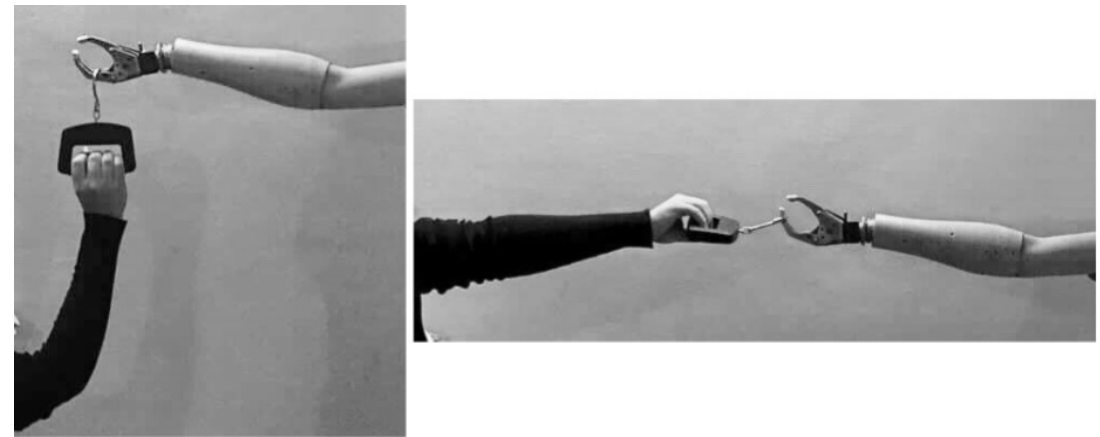

(a)

(b)

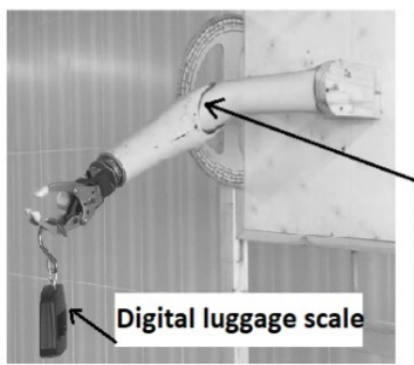

(c)

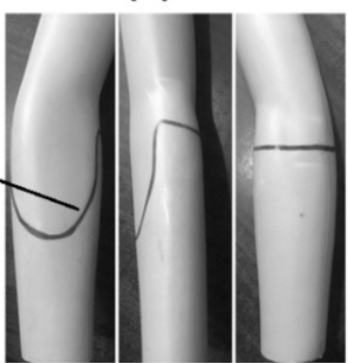

(d)

Figure 7: a) Live-Lift test; b) Axial-Load test; c) Female mannequin' arm simulating arm's anatomy of body human; and d) Mark on the female mannequin' arm.

during activities of daily living for the arm movements such as: Abduction and Adduction, Flexion and Extension, Supination and Pronation.

Experiment 1 (Live-lift test): The live-lift test measures the amount of vertical downward force applied at the socket that an amputee can resist without occurring dislocation between the residual limb and the socket while maintaining his arm at 90 deg as shown in Figure 7a [10].

Experiment 2 (Axial load test): The axial load 
test measures the amount of vertical downward force applied at the socket that amputee can resist without occurring dislocation between the residual limb and the socket while maintaining his arm in an extended position as shown in Figure $7 b[10]$.

The Digital luggage scale is used to measure the maximum force the socket can endurance. It has 50 $\mathrm{kg}$ capacity and Accuracy of up to $0.02 \mathrm{lb} / 10 \mathrm{~g}$ and 1 degree Celsius as shown in Figure 7c.

Special considerations: The Female mannequin' arm is used to simulate arm's anatomy of body human. The Mannequin residual limb model is fitted firmly with the socket and both were suspended from a wooden connection part as shown in Figure $7 c$.

To control successfully on tests and results, the socket must be donned firmly with a good degree of accuracy. So, we make a mark on the stump of the arm where the maximum fitted position is between it and the socket to be a guide for every time we change the position of the test as shown in Figure $7 d$.

\section{Results}

In Table 1, it shows that the live-lift force is larger than axial-load force at abduction case. In ad- duction case when angle is $85^{\circ}$, live-lift force larger than axial-load force also.

In Table 2, it shows that the live-lift force is larger than the axial-load force at flexion case when angle is $20^{\circ}$. In Extension case when angle is $175^{\circ}$, live- lift force is larger than axial-load force also.

In Table 3, In the pronation case, the live-lift force is larger than the axial-load force. In the supination case, the live-lift force is larger than the axial-load force also.

In Table 4, illustrates the flexion extension range of motion for the previous tests and the abilities of the introduced test rig.

\section{Discussion}

The test rig designs for of the lower limb sockets assessment are too many compared with the upper limb amputations. There are rarity in researches that assess the dislocation between the socket and the residual limb for the upper limb amputations. Accordingly, the study introduced the test rig design for the upper limbs to assess the socket as the lower limbs.

The test rig design depended on simulating the human arm movements. All parts of this design are made from wood. It is supplied with special parts to allow movements in Euler planes.

Table 1: Live-Lift and Axial-Load test for adduction and abduction movements.

\begin{tabular}{|l|l|l|l|}
\hline & Angle (degrees) & Live-Lift force (g) & Axial-Load force (g) \\
\hline Abduction & $0^{\circ}$ & 6325 & 3620 \\
\hline Adduction & $85^{\circ}$ & 2330 & 523 \\
\hline
\end{tabular}

Table 2: Live-Lift and Axial-Load test for flexion and extension movements.

\begin{tabular}{|l|l|l|l|}
\hline & Angle (degrees) & Live-Lift force (g) & Axial-Load force (g) \\
\hline Flexion & $20^{\circ}$ & 6577 & 3620 \\
\hline Extension & $175^{\circ}$ & 875 & 523 \\
\hline
\end{tabular}

Table 3: Live-Lift and Axial-Load test pronation and supination movement.

\begin{tabular}{|l|l|l|l|}
\hline & Angle (degrees) & Live-Lift force (g) & Axial-Load force (g) \\
\hline Pronation & $0^{\circ}$ & 6325 & 3620 \\
\hline Supination & $360^{\circ}$ & 5425 & 2752 \\
\hline
\end{tabular}

Table 4: Comparison of range of motion (ROM) between previous sockets and the socket of this study.

\begin{tabular}{|l|l|l|l|}
\hline $\begin{array}{l}\text { Comparison of ROM of } \\
\text { previous for Axial-Load }\end{array}$ & $\begin{array}{l}\text { Conventional supracondylar } \\
\text { suspension [8] }\end{array}$ & $\begin{array}{l}\text { Roll-on Sleeve } \\
\text { suspension [8] }\end{array}$ & Socket of this study \\
\hline ROM (degrees) & $30-110$ & $5-110$ & $20-175$ \\
\hline
\end{tabular}


Four pins are used to make movements in pitch and yaw angle. When two pins are connected with plate on the $\mathrm{Y}$-axis and be free with $\mathrm{X}$-axis, plate can rotate about the $Y$-axis and making yaw angle. Meanwhile, the plate rotates about $\mathrm{X}$-axis and making pitch angle when pins are connected only with plate on the X-axis. The wooden connection part is responsible for rotation the arm about Z-axis and making roll angle.

The purpose of this design is aid for assessment the used commercial socket experimentally. The shape of the socket and cross sections at different locations are elliptical with different diameters. The study implemented two tests for the socket (Live-Lift Test and Axial-Load Test). The two tests were done at different positions for human arm movements (Abduction, Adduction, Flexion, Extension, Supination and Pronation).

The socket was suspended on mannequin arm without considering a material that replicates the soft tissues of the human body. The results of experimental work of this study are not based on the interface between the socket and residual limb in vivo. In the future, materials such as Ballistic gel or silicone will be used to simulate the residual limb of a person with an amputation and test how the socket will be dislocated from the stump.

The certain angles that were selected in the experimental work expressed the maximum angles which perform by the test rig and predict when the dislocation between the socket and residual limb will occure. The tested socket didn't reach to the extremes $90^{\circ}$ and $180^{\circ}$ due to the edges of the test rig that restrict the arm movement.

Referring to the experimental work, the live-lift force is larger than the axial-load force in all cases (Abduction, Adduction, Flexion, Extension, Supination and Pronation). In pronation and supination cases, the variation of the measured forces is affected by the ending shape of the socket. In flexion and extension cases, the experimental tests are restricted by the edge of plate form of the test rig.

\section{Conclusion and Future Work}

The study introduced the test rig design to assess the commercial socket at different positions and planes that simulate the arm movements. The experimental work covers two main tests to assess the dislocation of the socket. Ultimately, this test rig design may aid in the development of the sockets and improve the quality of the design. Also, it helps to fulfill the design considerations for the socket without any suffering to the amputees.

In the future, the study could use special materials to resemble the soft tissues of the human body.

\section{References}

1. CH Jang, HS Yang, HE Yang, SY Lee, JW Kwon, et al. (2011) A survey on activities of daily living and occupations of upper extremity amputees. Ann Rehabil Med 35: 907-921.

2. Y Sang, $X$ Li, Y Luo (2016) Biomechanical design considerations for transradial prosthetic interface: A review. Proc Inst Mech Eng H 230: 239-250.

3. T Bertels, T Kettwig (2011) Breathable liner for transradial prostheses. Proceedings of the Myoelectric Symposium.

4. Y Sang, X Li, Y Gan, D Su, Y Luo (2014) A novel socket design for upper-limb prosthesis. International Journal of Applied Electromagnetics and Mechanics 45: 881-886.

5. Y Sang, X Li, Y Luo (2016) Characteristics of a volume-adjustable compression chamber for transradial prosthetic interface. Proc Inst Mech Eng H 230: 650-660.

6. JE Uellendahl, S Mandacina, S Ramdial (2006) Custom silicone sockets for myoelectric prostheses. Journal of Prosthetics and Orthotics 18: 35-40.

7. C Lake (2008) The evolution of upper limb prosthetic socket design. Journal of Prosthetics and Orthotics 20: 85-92.

8. WK Daly (2002) Electrodes installed in roll-on suspension sleeves. MyoElectric Controls/Powered Prosthetics Symposium Fredericton.

9. MM Wernke (2014) Quantification of transhumeral prosthetic socket residual limb interface movement using motion capture and a slip detection sensor. University of South Florida, Scholar Commons, USA.

10.MD Paskett, NR Olsen, JA George, DT Kluger, MR Brinton, et al. (2019) A modular transradial bypass socket for surface myoelectric prosthetic control in non-amputees. IEEE Trans Neural Syst Rehabil Eng 27: 2070-2076.

11.L Haverkate, G Smit, D Plettenburg (2014) Assessment of body-powered upper limb prostheses by able-bodied subjects, using the box and blocks test and the nine-hole peg test. Prosthet Orthot Int 40: 109-116. 
12.HW Wevers, JP Durance (1987) Dynamic testing of below-knee prosthesis: Assembly and components. Prosthet Orthot Int 11: 117-123.

13.WCC Lee, M Zhang, PPY Chan, DA Boone (2006) Gait analysis of low-cost flexible-shank transtibial prostheses. IEEE Trans Neural Syst Rehabil Eng 14: 370377.
14.SM Abbas, MH Abbas (2018) Analysis and manufacturing of above knee prosthesis socket by using revo fit solution. International Conference on Materials Engineering and Science 454: 012025.

15.S Fishman, HW Kay (1964) The munster-type below-elbow socket, an evaluation. Artificial Limbs 8: 4-14. 